\title{
BMJ Open Filling the gaps in SARDs research: collection and linkage of administrative health data and self-reported survey data for a general population-based cohort of individuals with and without diagnoses of systemic autoimmune rheumatic disease (SARDs) from British Columbia, Canada
}

To cite: McCormick N, Reimer K, Famouri A, et al. Filling the gaps in SARDs research: collection and linkage of administrative health data and self-reported survey data for a general population-based cohort of individuals with and without diagnoses of systemic autoimmune rheumatic disease (SARDs) from British Columbia, Canada. BMJ Open 2017;7:e013977. doi:10.1136/ bmjopen-2016-013977

- Prepublication history and additional material are available. To view these files please visit the journal online (http://dx.doi.org/ 10.1136/ bmjopen-2016-013977)

Received 22 August 2016 Revised 23 March 2017 Accepted 26 April 2017

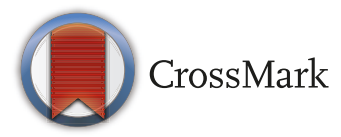

For numbered affiliations see end of article.

Correspondence to J. Antonio Aviña-Zubieta; azubieta@arthritisresearch.ca

\section{ABSTRACT}

Purpose Systemic autoimmune rheumatic diseases (SARDs) are a group of debilitating autoimmune diseases, including systemic lupus erythematosus and related disorders. Assessing the healthcare and economic burden of SARDs has been challenging: while administrative databases can be used to determine healthcare utilisation and costs with minimal selection and recall bias, other health, sociodemographic and economic data have typically been sourced from highly selected, clinic-based cohorts. To address these gaps, we are collecting selfreported survey data from a general population-based cohort of individuals with and without SARDs and linking it to their longitudinal administrative health data.

Participants Using administrative data from the province of British Columbia (BC), Canada, we established a population-based cohort of all $\mathrm{BC}$ adults receiving care for SARDs during 1996-2010 $(n=20729)$ and non-SARD individuals randomly selected from the general population. BC Ministry of Health granted us contact information for 12000 SARD and non-SARD individuals, who were recruited to complete the surveys by mail or online.

Findings to date Four hundred individuals were initially invited to participate, with 135 (34\%) consenting and 127 (94\%) submitting the first survey (72\% completed online). Sixty-three $(49.6 \%)$ reported $\geq 1$ SARD diagnosis. The non-SARDs group $(n=64)$ was $92 \%$ female with mean age $57.0 \pm 11.6$ years. The SARDs group ( $n=63$ ) was $94 \%$ female with mean age $56.5 \pm 13.1$ years. Forty-eight per cent of those with SARDs were current-or-former smokers (mean $10.6 \pm 16.2$ pack-years), and $33 \%$ were overweight or obese (mean body mass index of 24.4 \pm 5.3 ).

Future plans Health and productivity data collected from the surveys will be linked to participants' administrative

\section{Strengths and limitations of this study}

- This is the first population-based cohort of systemic autoimmune rheumatic diseases (SARDs) in Canada, and one of the first Canadian endeavours to link administrative health data with prospectively collected, self-reported survey data from any disease group, let alone SARDs.

- Access to administrative health data from a singlepayer healthcare system allowed us to establish a population-based cohort of individuals with and without SARDs, which should increase the generalisability of research results.

- Collecting survey data from a random sample of this cohort, and linking it to their administrative data, will allow us to determine more components of the economic burden of SARDs (direct medical costs, out-of-pocket costs and productivity losses from paid and unpaid work) and identify more predictors of health resource utilisation, direct medical costs and other health outcomes than is possible when using administrative health databases alone.

- However, the representativeness of the survey sample will ultimately depend on which of the invited individuals actually complete the surveys.

- While the response rate from our randomly selected sample (34\%) was encouraging, further approaches to increase response rates are needed.

health data from the years 1990-2013, allowing us to determine the healthcare and lost productivity costs of SARDs, and assess the impact of patient-reported variables on utilisation, costs, disability and clinical outcomes. Findings will be disseminated through scientific conferences and peer-reviewed journals. 


\section{INTRODUCTION}

Systemic autoimmune rheumatic diseases (SARDs) are a group of chronic arthritides including systemic lupus erythematosus (SLE), Sjogren's syndrome, systemic sclerosis/scleroderma, polymyositis and dermatomyositis and forms of adult systemic vasculitis (eg, giant cell arteritis and granulomatosis with polyangiitis). Immune dysregulation in SARDs leads to systemic inflammation, organ damage, a high morbidity burden and reduced health-related quality of life. ${ }^{1-4}$ The physical and cognitive manifestations of SARDs can also limit patients' participation in paid employment, ${ }^{5-12}$ family life ${ }^{13} 14$ and other meaningful activities. ${ }^{15}$ Though distinct disorders, SARDs are often studied together due to their shared pathogenesis, manifestations and treatments, which consist mainly of glucocorticoids and other immune-modulating and immunosuppressive therapies.

As SARDs are relatively rare, collectively affecting approximately $0.2 \%-0.5 \%$ of Canadian adults, ${ }^{16}$ the healthcare, economic and societal burden imparted by these diseases is largely unknown. Economic burden includes direct medical costs (costs for the provision of healthcare resources like outpatient encounters, hospitalisations and medications) and costs associated with lost productivity. To date, most estimates of direct medical costs for SARDs have pertained only to SLE and were determined over short periods of time from small numbers of patients at tertiary care centres. Such patients tend to have more severe disease, which reduces the generalisability of these cost estimates. Moreover, tertiary care centres and disease registries that collect data on SARDs typically do not collect comparable information on people without SARDs. This makes it difficult (if not impossible) to determine the incremental burden of these disorders or cost drivers specific to SARDs. Estimates of productivity losses in SARDs and the associated costs are similarly lacking, with the majority of estimates pertaining to SLE, and sourced from highly selected, clinic-based samples. Furthermore, most have focused on the costs associated with just one aspect of lost productivity, absenteeism from paid employment and have not considered absenteeism from unpaid work or reduced productivity (presenteeism) while undertaking paid or unpaid work.

Canadian administrative databases are a rich source for determining direct medical costs at the population level since they capture the health resource utilisation of nearly every resident in a universal healthcare system. However, these databases do not contain information on the direct medical costs of services not funded by the province (ie, non-prescription medications, complementary care or most visits to allied health professionals), direct non-medical costs (ie, parking or travel costs incurred by patients while obtaining medical care) or lost productivity costs. Furthermore, they lack variables on sociodemographics and health behaviours that may be predictors of costs in themselves or can serve as covariates when assessing the impact of potential cost predictors. Such information is best collected from individuals directly; however, given the rarity of SARDs, recruiting a representative sample to provide this information is difficult.

To address these limitations, we established population-based SARD and matched non-SARD cohorts from the administrative health databases of one Canadian province, British Columbia (BC), and are inviting a sample of the cohort to complete a health research survey. Survey data on participants' productivity losses, out-of-pocket costs, health status, sociodemographics and health and lifestyle behaviours will be linked with their administrative health data. This will allow us to quantify the economic burden of SARDs at the population level more comprehensively than if using administrative health databases alone.

\section{COHORT DESCRIPTION/METHODS \\ Data source}

Publicly funded healthcare is available to all residents of the province of British Columbia (BC), Canada (population 4.5 million). Population Data BC uses population-based linkable administrative data files to capture all provincially funded healthcare services, including all outpatient medical visits (to specialists and primary care physicians), interventions, investigations ${ }^{17}$ cancer diagnoses, ${ }^{18}$ and hospital admissions and discharges ${ }^{19}$ since 1990 , as well as limited demographic ${ }^{20}$ and vital statistics data. ${ }^{21}$ Up to five diagnoses are recorded for each outpatient encounter and up to 25 diagnoses for each inpatient admission. Furthermore, it encompasses the comprehensive prescription drug database PharmaNet, ${ }^{22}$ which includes all dispensed medications for all $\mathrm{BC}$ residents regardless of age or funding source. Numerous general population-based studies have been successfully conducted using these databases. ${ }^{23-26}$

\section{Population-based cohorts}

From the administrative data files, we assembled a population-based cohort of all BC adults with SARDs during the years 1996-2010. SARDs cases were identified on the basis of International Classification of Diseases Ninth (ICD-9) and Tenth (ICD-10) Revision diagnostic codes (see online supplementary file) recorded for outpatient encounters and hospitalisations, having either: (A) $\geq 2$ ICD-9 codes for SARDs $\geq 2$ months apart but within a 2-year period by a non-rheumatologist physician; or (B) one ICD-9 or ICD-10 code for SARDs by a rheumatologist (at any time) or from hospitalisation.

The validity of this case definition has been evaluated in a Canadian context, wherein the sensitivity for most SARDs was $\geq 88 \%$ and the specificity was $\geq 95 \% .{ }^{27}$ Nevertheless, to further improve specificity, we excluded potential cases with at least two visits $\geq 2$ months apart (subsequent to the SARD index visit) with diagnoses of other inflammatory arthritides, including rheumatoid arthritis, psoriatic arthritis and spondyloarthropathies. To establish the non-SARD comparison cohort, we received data for a random sample of $400000 \mathrm{BC}$ residents (those 
registered with the provincial medical services plan any time during the study period). From these data, up to 10 individuals without a SARD diagnosis were matched to each case based on age, sex and calendar year of study entry.

\section{Survey sample}

The administrative health data files are released to researchers in a de-identified form, stripped of any names, addresses or phone numbers that would allow researchers to identify or contact these individuals. However, there is a new process in $\mathrm{BC}$ where researchers may apply for access to the names and contact information of a sample of individuals in administrative health databases, for the sole purpose of recruiting them to participate in a specific health research study. This 'Request-to-Contact' application must be approved by a number of governing bodies, including the institutional research ethics board, the BC Ministry of Health's Data Stewardship Committee and the Office of the Information and Privacy Commissioner for BC.

On receiving final approval for our Request-to-Contact application from all governing bodies in April 2015, we assembled a subset of the SARD and comparison cohorts. Included in the subset were 9335 prevalent SARD cases (82.3\% female, mean age (SD) in 2015 of 60 (15.8) years) and 55431 matching non-SARD individuals $(82.8 \%$ female, mean age 62.4 (16.0) years). The BC Ministry of Health selected a random sample $(n=12000)$ of these individuals, and in July 2015, their names, addresses and phone numbers were released to the research team. Although 6000 of these individuals were selected because they had a SARD diagnosis recorded in the administrative databases, and 6000 were selected because they did not have a SARD diagnosis, the information on all 12000 individuals was provided in a single file, and the research team was blinded to the disease status of each individual.

\section{Recruitment}

Our recruitment strategy was developed using the Dillman method $^{28}$ and adapted, where necessary, to meet Office of the Information and Privacy Commissioner regulations concerning the number of contacts that could be made and timing and format of each contact. Potential participants were mailed an invitation package complete with a personalised letter of invitation from the principal investigator, a separate invitation from the BC Ministry of Health, two copies of the consent form, and an addressed, prepaid envelope for returning one copy of the consent form. Included in the invitation letters and consent form were a description of the study, and how and why the recipient's name and contact information were disclosed to the research team. Potential participants were told that the study involved completing two short surveys on their paid and unpaid work activities. We emphasised the importance of having both people with and without SARDs participate and described the measures in place to protect the privacy of each participant's personal information, including their disease status.
A \$2 coin was included in the invitation package as a token of appreciation for the recipient's time and an incentive to participate. Small tokens (between $\$ 1$ and $\$ 5$ ) that are delivered as part of the initial request to respond to mail questionnaires have been found to increase response rates, ${ }^{29}$ whereas promises of tokens on completion are less effective. ${ }^{28}{ }^{30}$ All contacted individuals, regardless of whether they chose to participate in the study, were given the option of keeping the $\$ 2$, or mailing it back to the research team for donation to one of five SARD-related charities of their choice. As an additional incentive, participants who complete both surveys will be entered into a draw to win 1 of $10 \$ 100$ cash prizes and one $\$ 1000$ grand prize.

Individuals who wished to participate in the study were asked to review and sign the consent form, return one copy to the research team and retain the other copy for their records. The form asked participants to indicate whether they wanted to complete the surveys online or on paper and whether the research team could contact them at a later time about using their survey data in future analyses.

Potential participants who did not respond to the invitation package after 2 weeks were mailed a reminder letter. Four weeks after the invitation package was mailed, a member of the research team was permitted to make up to two attempts to contact non-respondents by phone. Being able to reach potential participants by phone was critical as some individuals had moved (such that their address on file with the BC Ministry of Health was out-of-date) but could still be reached at the same phone number. If, when reached by phone, such individuals expressed interest in the study, another invitation package was mailed to them at their new address.

\section{Data collection and analysis}

The first survey was distributed on receipt of the completed consent form. Participants completing the surveys online were emailed a link to the first survey, along with a unique six-digit username that allowed them to complete the survey over multiple sessions and save their responses as they went along. Participants who requested paper surveys were mailed a copy of the survey along with a prepaid return envelope. All participants were asked to complete and submit the survey within 2 weeks of receipt. Participants who did not submit the online survey within this time were sent an email reminder, while those who did not return a paper survey were sent a reminder letter by mail. As requested by the Data Stewards, the reminders included explicit instructions on how participants could withdraw from the study, if they so desired. If participants did not return the survey within 4 weeks, a member of the research team followed up with them by phone.

The same data collection and follow-up procedures will be employed with the second survey. Once collected and cleaned, survey data will be used to determine the lost productivity and out-of-pocket costs of SARDs. It will subsequently be linked to participants' administrative 
health data, allowing us to assess how key self-reported sociodemographic and clinical variables are associated with health resource utilisation and direct medical costs.

\section{Survey components}

Each of the two surveys for this study are self-administered and comprise six sections. The second survey is nearly identical to the first but does not ask about sociodemographic factors that would not (or are unlikely) to change over time, such as gender or educational attainment. The surveys and other study documents were reviewed and pilot-tested by research staff at Arthritis Research Canada and volunteer members of our SARDs Consumer Advisory Council. Through this pilot-testing, we estimated that the first survey could be completed in $30 \mathrm{~min}$, and the second, shorter, follow-up survey in $15 \mathrm{~min}$.

- Section One: using questions from the Canadian Community Health Survey ${ }^{31}$ and other established health research questionnaires, data are collected on sociodemographic variables (ie, marital status, race and educational attainment), health exposures and behaviours (ie, height, weight, smoking history, alcohol use and exercise), diagnoses of SARDs and any other forms of arthritis and selected comorbid conditions, month and year of SARD diagnosis by a health professional (and start of symptoms), use of health resources not captured in the provincial administrative databases (ie, non-prescription medications, complementary care, medical devices and visits to allied health professionals) and out-of-pocket costs.

- Section Two: the EQ-5D-5L instrument ${ }^{32} 33$ is used to collect data on health-related quality-of-life. This instrument asks respondents to rate five separate dimensions of their health on that particular day: mobility, self-care, usual activities, pain/discomfort and anxiety/depression. Respondents are also asked to rate their current health on a 100-point scale.

- Section Three: data are collected on health status, including level of disability (using the Health Assessment Questionnaire Disability Index ${ }^{34}$ ) and severity of pain and fatigue over the past 7 days (using a 100-point visual analogue scale).

- Sections Four and Five: data on lost productivity are collected using the Work Productivity and Activity Impairment (WPAI) Questionnaire ${ }^{35}$ in Section 4, and Valuation of Lost Productivity (VOLP) ${ }^{36}$ questionnaire in Section Five. Two productivity questionnaires are included because, although the WPAI is fairly short (maximum of six questions presented over two pages), it only allows for the valuation of productivity losses from paid employment. The VOLP, a newer questionnaire that has been validated in a population of rheumatoid arthritis patients ${ }^{37}$ and used to assess the productivity costs of rheumatoid arthritis ${ }^{38}$ and asthma, ${ }^{39}$ allows for the valuation of productivity losses (absenteeism and presenteeism) from both paid and unpaid activities. It also considers the productivity losses experienced by colleagues when one member of the team is away from work or less productive. Included in the second survey are questions about change in employment status since the first survey.

- Section Six: participants are asked if they are interested in receiving information about the results of the study and about future health research studies conducted at The University of British Columbia. This will facilitate the establishment of a population-based SARD cohort and comparison group for long-term study, although individuals will need to provide additional consent before participating in any future research.

\section{Privacy and confidentiality}

A number of measures were enacted to ensure the privacy of potential participants' personal information and the security of data throughout the recruitment, data collection and analysis processes. All electronic information, including names, contact information and survey data, will remain in Canada, housed in encrypted, password-protected computerised files on secured network servers. Participants will only be identified on study documents by a survey ID number; personal information (ie, names, contact information) will not be linked to survey results, nor administrative health data, at any time.

It was also important to ensure that an individual's disease status would not be unwittingly revealed to the research team or other individuals during the recruitment process. To prevent this, the invitation letter was carefully worded to communicate that the individual had been randomly selected to participate in the study as someone who 'may or may not' have been diagnosed with a SARD. Furthermore, when leaving phone messages for potential participants, we identified the study only as a 'health research study conducted at The University of British Columbia', without mention of the specific diagnoses under study.

\section{Findings to date}

After receiving the names and contact information of the potential participants on 9 July 2015, we began recruitment by conducting two pilot mailouts of 200 invitation packages each. The first mailout was conducted almost immediately, and the second was conducted in early September, in order to see if the recruitment process would be different outside of the summer months. To date, from the two mailouts combined, 135 individuals have consented to participate, for a consent rate of $33.8 \%$. The consent rate from the first (summer) mailout was slightly higher than the second $(36.5 \%$ (73 consents) versus $31 \%$ (62 consents), respectively).

Figure 1 is a flowchart of the recruitment process. Of the 265 who did not consent, 115 formally indicated their refusal to participate. Thirty-seven per cent of these individuals did not provide a reason, while $21 \%$ said they were too busy, $18 \%$ cited advanced age or health problems, $14 \%$ said they lacked fluency in English, $4 \%$ expressed privacy concerns, $3 \%$ said they were not 


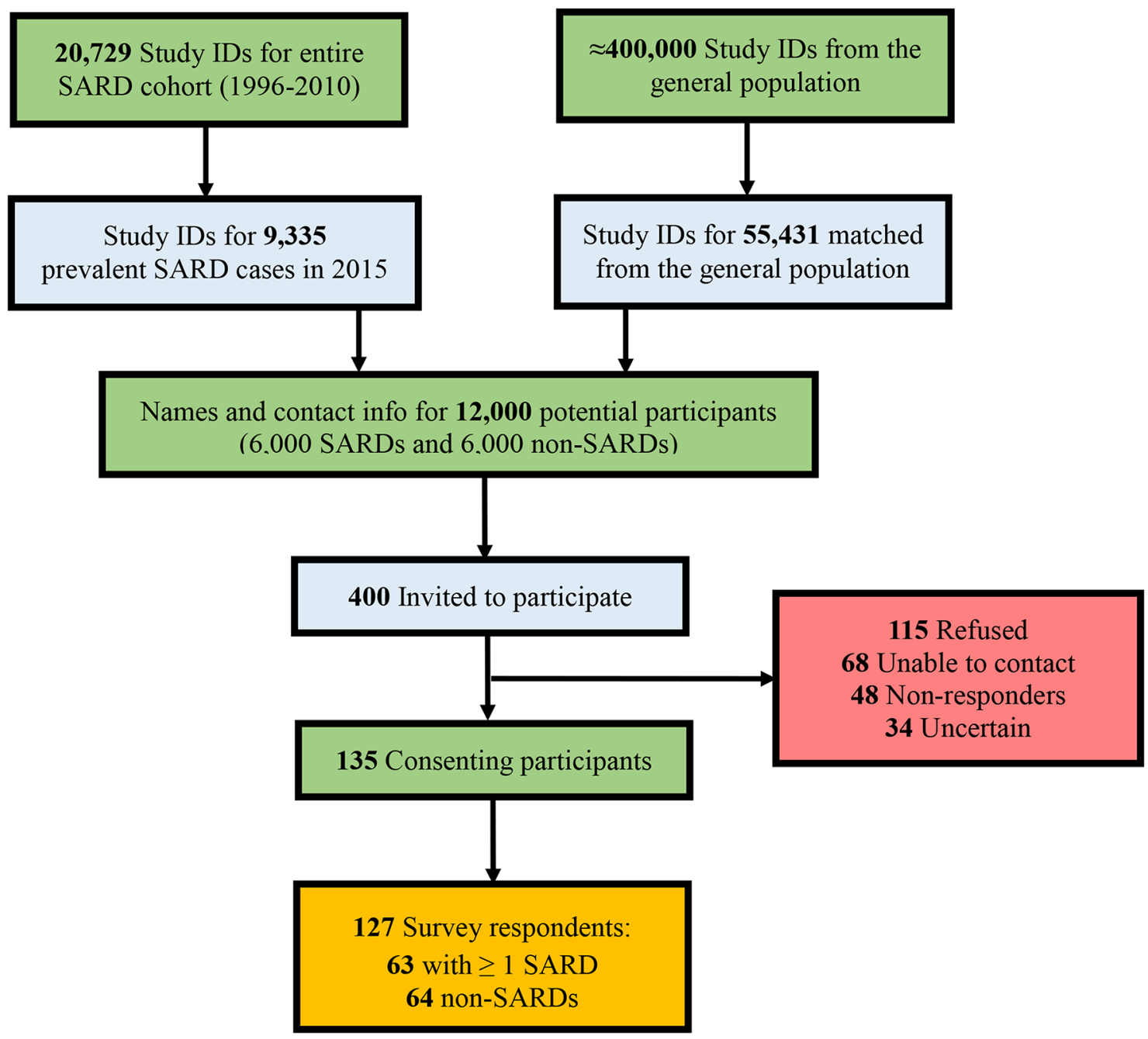

Figure 1 Recruitment flowchart. SARDs, systemic autoimmune rheumatic diseases.

interested and 3\% had moved out of the province or died. We classified $68(17 \%)$ of the remaining individuals as 'Unable-to-Contact' (ie, incorrect address, phone number out-of-service or phone line constantly busy), 48 $(12 \%)$ as 'Non-Responders', meaning that two messages (the maximum allowed by the Office of the Information and Privacy Commissioner) were left on the individual's voicemail or answering machine with no return phone call or other form of response and $34(8.5 \%)$ as 'Uncertain', being individuals who have expressed interest in the study and may still return their consent form at a later date.

Sixty-nine out of the 400 who were sent a package (17\%) returned the $\$ 2$ coin for donation to charity, including 14 who declined to participate in the study. Of the 40 people who specified a charity, Arthritis Research Canada and the BC Lupus Society were the most frequent beneficiaries.

Thus far, $94 \%$ of consenting participants have successfully submitted their first survey, with nearly three-quarters $(\mathrm{n}=91 / 127)$ completed online, and $61 \%$ agreeing to be contacted about future studies. Sixty-three participants $(49.6 \%)$ reported at least 1 SARD diagnosis including
33 SLE, 17 Sjogren's syndrome, 6 systemic sclerosis, 3 polymyositis, 4 dermatomyositis, 2 granulomatosus with polyangiitis and 7 giant cell arteritis. Baseline characteristics of the SARD and non-SARD groups are shown in table 1 . The non-SARD individuals were $92 \%$ female with mean (SD) age 57.0 (11.6) years. Those with SARDs were $94 \%$ female with mean age 56.5 (13.1) years. The mean age at diagnosis of SLE was 33.3 \pm 11.1 years, Sjogren's syndrome was $45.1 \pm 14.0$ years and systemic sclerosis was $44.5 \pm 7.3$ years. The mean durations of disease (based on patients' self-reported month and year of diagnosis) were $17.4 \pm 7.3,11.6 \pm 9.5$ and $11.5 \pm 8.8$ years, for SLE, Sjogren's syndrome and systemic sclerosis, respectively.

\section{Smoking and body mass index (BMI)}

As one of our key analyses will be to examine the impact of cigarette smoking, BMI and overweight and obesity on health resource use, work disability and costs in SARDs, we sought to determine the smoking history and BMI of our cohorts. Almost equal proportions of the SARD and non-SARD cohorts had ever smoked (48\% of SARDs and $45 \%$ of non-SARDs), and data on pack-years of smoking (years of smoking*number of packs smoked per day) 
Table 1 Baseline characteristics for SARDs and non-SARDs cohorts

\begin{tabular}{|c|c|c|c|}
\hline & SARDs & Non-SARDs & p Value \\
\hline $\mathrm{N}$ & 63 & 64 & - \\
\hline N Female (\%) & $59(94)$ & $59(92)$ & 0.75 \\
\hline Mean age in 2015 (SD) & $56.5(13.1)$ & $57.0(11.6)$ & 0.89 \\
\hline Median age in 2015 (IQR, range) & $57(20)$ & $58(14.5)$ & \\
\hline $\mathrm{N}$ with partner (\%) & $42(67)$ & $47(73)$ & 0.41 \\
\hline $\begin{array}{l}\mathrm{N} \text { with children at home (\%) } \\
(n=5 \text { missing responses) }\end{array}$ & $21(33)$ & $23(36)$ & 0.76 \\
\hline $\mathrm{N}$ with high school education or less (\%) & $17(27)$ & $23(36)$ & 0.28 \\
\hline $\mathrm{N}$ completed university (\%) & $21(33)$ & $20(31)$ & 0.80 \\
\hline N Current or Former Smokers (\%) & $30(48 \%)$ & $29(45 \%)$ & 0.79 \\
\hline Mean pack-years, among ever-smokers (SD) ( $n=3$ missing responses) & $10.6(16.2)$ & $25.2(36.0)$ & 0.06 \\
\hline Mean years of smoking, among ever-smokers (SD) ( $n=1$ missing response) & $16.7(13.7)$ & $24.2(13.4)$ & 0.04 \\
\hline $\begin{array}{l}\text { Mean years since smoking cessation, among former smokers (SD) } \\
\text { ( } n=3 \text { missing responses) }\end{array}$ & $16.6(13.6)$ & $17.3(8.4)$ & 0.84 \\
\hline $\mathrm{N}$ consumed alcohol in past 12 months (\%) & $47(75)$ & $49(77)$ & 0.79 \\
\hline Mean body mass index (BMI) (SD) & $24.4(5.3)$ & $27.0(6.4)$ & 0.01 \\
\hline N overweight $(\%)\left(B M I \geq 25 \mathrm{~kg} / \mathrm{m}^{2}\right)$ & $21(33)$ & $35(55)$ & 0.02 \\
\hline N obese $(\%)\left(B M I \geq 30 \mathrm{~kg} / \mathrm{m}^{2}\right)$ & $9(14)$ & $21(33)$ & 0.01 \\
\hline Mean BMI, corrected ${ }^{\star}(\mathrm{SD})$ & $25.5(5.6)$ & $28.1(6.8)$ & 0.02 \\
\hline N overweight, after correction* (\%) & $25(40)$ & $37(58)$ & 0.04 \\
\hline N obese, after correction $\left.{ }^{*} \%\right)$ & $13(21)$ & $24(38)$ & 0.04 \\
\hline
\end{tabular}

${ }^{*}$ Correction equations ${ }^{40}$ were applied to the original height and weight data to account for the fact that individuals tend to overestimate their height, and underestimate their weight, in self-reports.

SARDs, systemic autoimmune rheumatic diseases.

were available from 29 of 30 SARD smokers, and 27 of 29 non-SARD smokers. Among these individuals, there was a tendency (though non-significant) for those without SARDs to have a heavier smoking history than those with SARDs (mean pack-years of 25.2 (36.0) and 10.6 (16.2), respectively, $\mathrm{p}=0.06$

Despite only having data for 127 individuals, we did observe statistically significant differences in the mean BMI, and the proportion of overweight (BMI $\geq 25 \mathrm{~kg} / \mathrm{m}^{2}$ ) and obesity (BMI $\geq 30 \mathrm{~kg} / \mathrm{m}^{2}$ ), between the SARD and non-SARD cohorts. Those without SARDs averaged a significantly higher BMI than those with SARDs (27.0 vs 24.4, $\mathrm{p}=0.01$ ), and a greater proportion of non-SARDs were both overweight ( $55 \%$ vs $33 \%, \mathrm{p}=0.02)$ and obese $(33 \%$ vs $14 \%$, $\mathrm{p}<0.01)$. Knowing that individuals tend to overestimate their height in self reports and underestimate their weight, ${ }^{41-43}$ we recalculated BMI after applying sex-specific correction equations for self-reported height and weight that have been developed for the Canadian population. ${ }^{40}$ While this correction increased the mean BMI of both groups slightly (table 1), non-SARDs continued to have a significantly higher BMI than those with SARDs and were more likely to be overweight or obese.

\section{Strengths and limitations}

The strengths and limitations of our study design deserve comment. One strength is the selection of participants with and without SARDs from a population-based sample of 4.5 million people, allowing our results to be generalisable at the population level. We expect that recruiting a random sample of these individuals to complete the survey will minimise selection bias and make for a cohort that is more representative of different sociodemographic backgrounds and disease severities. However, the representativeness of our survey cohort will ultimately depend on which of the invited individuals actually consent to participate and complete the surveys. If those who consent differ in their health resource use and productivity losses from those who do not, the generalisability of our findings will be reduced. As our SARD cohort includes a mix of incident and prevalent cases with rather established disease (mean disease duration of $17.4 \pm 7.3$ years for SLE and 11.5 \pm 8.8 years for systemic sclerosis), we will be limited in our ability to assess the costs of newly diagnosed cases.

Another concern is our ability to recruit individuals who do not have a SARD diagnosis, since they will not have the same motivation to participate as those with SARDs. With this in mind, we are making efforts to ensure a broad range of individuals will participate. For example, we are providing the option of paper surveys to encourage participation from those who do not have access to a computer or who are less comfortable using computers. 
Participants may complete the surveys at their own time and pace and do not have to partake in visits or telephone interviews, which should appeal to those with unpredictable work or school schedules, family responsibilities or health issues. As well, those who wish to complete the surveys in languages other than English, including Chinese, Punjabi, French and Spanish may do so with the assistance of a member of the study team who speaks their preferred language. However, in spite of our efforts to recruit individuals across a range of disease severities and functional abilities, the participatory nature of this study precludes us from collecting data from those who are too frail, or too ill, to provide consent or complete the survey. Distributing the invitation packages to potential participants with incorrect addresses is also a challenge.

While the survey allows us to collect data on potential determinants of costs and health outcomes that are not available in the administrative data, the validity of some of these self-reported data may be suboptimal. However, Canadian research does support the validity of self-reported information on smoking. ${ }^{44}$ Finally, although the labour questionnaires used in our surveys (WPAI and VOLP) are generic instruments, we acknowledge that neither have been formally validated in SARDs. Still, both the $\mathrm{WPAI}^{45}$ and $\mathrm{VOLP}^{37}$ have been validated in populations of rheumatoid arthritis, and the WPAI has been used previously to measure productivity losses in SLE. ${ }^{46}$

\section{COLLABORATION}

We are open to collaboration with other researchers and/or consumer groups interested in investigating the disease, healthcare, economic and societal burden of SARDs. If you are interested, please contact the principal investigator, Dr Aviña-Zubieta. Please note that any data analysis must be conducted in Canada, in collaboration with the original investigators, on their secure servers. Such analysis is subject to approvals from the institutional research ethics boards and the Data Stewardship Committee of the BC Ministry of Health. Further consent from the study participants may also be required.

\section{FURTHER DETAILS}

This is, to our knowledge, one of the first health research endeavours in Canada to link administrative health data with prospectively collected, self-reported survey data from any disease group, let alone SARDs. With a consent rate of $34 \%$ and completion rate of $94 \%$ (with $72 \%$ of surveys completed online), we believe the response to our initial mailouts supports the feasibility of this Requestto-Contact approach for recruiting population-based cohorts. We have since invited an additional 2000 individuals with and without SARDs to participate in our study and expect to continue recruitment through the summer of 2016 with the aim of enrolling another 500 participants.
Following recruitment and survey completion, data collected from the surveys will be linked to participants' administrative health data, allowing us to determine the direct medical costs, direct non-medical costs and lost productivity costs of SARDs and to assess the impact of self-reported variables (ie, educational attainment, BMI, smoking history, level of disability and health-related quality of life) on health resource utilisation, costs and clinical outcomes. We also plan to investigate how self-reported data collected from the survey subsample could be imputed for the entire SARD and non-SARD cohorts, which would greatly increase our statistical power. Finally, in the course of this work, we hope to build a pool of representative SARD and non-SARD individuals that we can recruit for future studies aimed at improving our understanding of these rare, devastating diseases.

\section{Author affiliations}

${ }^{1}$ Faculty of Pharmaceutical Sciences, The University of British Columbia, Vancouver, Canada

${ }^{2}$ Arthritis Research Canada, Richmond, Canada

${ }^{3}$ Department of Occupational Science \& Occupational Therapy, Faculty of Medicine, The University of British Columbia, Vancouver, Canada

${ }^{4}$ School of Pharmacy, University of Otago, Dunedin, New Zealand

${ }^{5}$ Division of Rheumatology, Department of Medicine, The University of British Columbia, Vancouver, Canada

Correction notice This paper has been amended since it was published Online First. Owing to a scripting error, some of the publisher names in the references were replaced with 'BMJ Publishing Group'. This only affected the full text version, not the PDF. We have since corrected these errors and the correct publishers have been inserted into the references.

Acknowledgements We are most grateful to the members of the SARDs Consumer Advisory Council at Arthritis Research Canada (Ms Lianne Gulka, Ms Janis McCaffrey, Ms Joyce Ma, Ms Marilyn Mulldoon and Mr Alan Pemberton) for their assistance in developing the recruitment procedures, surveys and other study materials. We would also like to thank Dr Jacek Kopec for his contributions to the survey design, Lindsay Belvedere for her editorial assistance in the preparation of this manuscript and Sophia Sidi and Shannon Percival Smith for their assistance with data collection, entry and coding. All inferences, opinions and conclusions drawn in this manuscript are those of the authors and do not reflect the opinions or policies of the data steward(s).

Contributors Conception and design of the study: CAM and JAA-Z. Acquisition of the data: JAA-Z. Analysis and interpretation of the data: NM, KR, AF, CAM and JAA-Z. Drafting of the manuscript: NM and KR. Critical revision of the manuscript for important intellectual content: AF, CAM and JAA-Z. All authors gave final approval of the submitted manuscript.

Funding This work was supported by the Canadian Arthritis Network (grant \# 10-SRP-IJD-01) and the Canadian Institutes of Health Research (grant \# THC-135235). NM is supported by a Doctoral Research Award from the Canadian Institutes of Health Research. JAA-Z is currently the British Columbia Lupus Society Scholar and holds a Scholar Award from the Michael Smith Foundation for Health Research. The funders had no role in study design, data collection and analysis, decision to publish or preparation of the manuscript.

Competing interests None declared.

Patient consent This submission does not contain personal medical information about an identifiable individual, living or dead.

Ethics approval Behavioural Research Ethics Board, University of British Columbia. Provenance and peer review Not commissioned; externally peer reviewed. Data sharing statement No additional data are available.

Open Access This is an Open Access article distributed in accordance with the Creative Commons Attribution Non Commercial (CC BY-NC 4.0) license, which permits others to distribute, remix, adapt, build upon this work non-commercially, and license their derivative works on different terms, provided the original work is 
properly cited and the use is non-commercial. See: http://creativecommons.org/ licenses/by-nc/4.0/

(C) Article author(s) (or their employer(s) unless otherwise stated in the text of the article) 2017. All rights reserved. No commercial use is permitted unless otherwise expressly granted.

\section{REFERENCES}

1. Jolly M. How does quality of life of patients with systemic lupus erythematosus compare with that of other common chronic illnesses? J Rheumatol 2005;32:1706-8.

2. Hudson M, Thombs BD, Steele R, et al. Quality of life in patients with systemic sclerosis compared to the general population and patients with other chronic conditions. J Rheumatol 2009;36:768-72.

3. Faurschou M, Sigaard L, Bjorner JB, et al. Impaired health-related quality of life in patients treated for Wegener's granulomatosis. $J$ Rheumatol 2010;37:2081-5.

4. Carpenter DM, Thorpe CT, Lewis M, et al. Health-related quality of life for patients with vasculitis and their spouses. Arthritis Rheum 2009;61:259-65.

5. Baker K, Pope J. Employment and work disability in systemic lupus erythematosus: a systematic review. Rheumatology 2009;48:281-4

6. Baker K, Pope J, Fortin P, et al. Work disability in systemic lupus erythematosus is prevalent and associated with socio-demographic and disease related factors. Lupus 2009;18:1281-8.

7. Al Dhanhani AM, Gignac MA, Su J, et al. Work disability in systemic lupus erythematosus. Arthritis Rheum 2009;61:378-85.

8. Hudson M, Steele R, Lu Y, et al. Work disability in systemic sclerosis. $J$ Rheumatol 2009;36:2481-6.

9. Ouimet JM, Pope JE, Gutmanis I, et al. Work disability in scleroderma is greater than in rheumatoid arthritis and is predicted by high $\mathrm{HAQ}$ scores. Open Rheumatol J 2008;2:44-52.

10. Campbell R, Cooper GS, Gilkeson GS. The impact of $s$ ystemic lupus erythematosus on employment. J Rheumatol 2009;36:2470-5.

11. Boomsma MM, Bijl M, Stegeman CA, et al. Patients' perceptions of the effects of systemic lupus erythematosus on health, function, income, and interpersonal relationships: a comparison with Wegener's granulomatosis. Arthritis Rheum 2002;47:196-201.

12. Mau W, Listing J, Huscher D, et al. Employment across chronic inflammatory rheumatic diseases and comparison with the general population. J Rheumatol 2005;32:721-8.

13. Poole JL, Willer K, Mendelson C. Occupation of motherhood challenges for mothers with scleroderma. Am J Occup Ther 2009;63:214-9.

14. Poole JL, Rymek-Gmytrasiewicz M, Mendelson C, et al. Parenting: the forgotten role of women living with systemic lupus erythematosus. Clin Rheumatol 2012;31:995-1000.

15. Katz $P$, Morris A, Trupin L, et al. Disability in valued life activities among individuals with systemic lupus erythematosus. Arthritis Rheum 2008;59:465-73.

16. Broten L, Aviña-Zubieta JA, Lacaille D, et al. Systemic autoimmune rheumatic disease prevalence in Canada: updated analyses across 7 provinces. J Rheumatol 2014;41:673-9.

17. British Columbia Ministry of Health creator 2013. Medical services plan (MSP) payment information file. population data BC (publisher). Data extract. MOH (2013). http://www.popdata.bc.ca/ data.

18. BC Cancer Agency Registry Data (2014). Population data BC (publisher) data extract. BC cancer agency 2013 http://www. popdata.bc.ca/data.

19. Canadian Institute for Health Information creator 2013. D ischarge abstract database (Hospital separations) population aata BC (publisher) data extract. MOH (2013). http://www.popdata.bc.ca/ data.

20. British Columbia Ministry of Health creator 2013. Consolidation file (MSP Registration \& Premium Billing). Population data BC (publisher). Data extract. MOH. 2013 http://www.popdata. bc.ca/data.

21. BC Vital Statistics Agency creator 2012. Vital statistics deaths. Population data BC (publisher) data extract BC vital statistics agency 2013 http://www.popdata.bc.ca/data.
22. BC Ministry of Health creator 2013. PharmaNet. BC Ministry of Health (publisher) Data extract. Data Stewardship Committee (2013). http://www.popdata.bc.ca/data.

23. Solomon DH, Massarotti E, Garg R, et al. Association between disease-modifying antirheumatic drugs and diabetes risk in patients with rheumatoid arthritis and psoriasis. JAMA 2011;305:2525-31.

24. Aviña-Zubieta JA, Abrahamowicz M, Choi HK, et al. Risk of cerebrovascular disease associated with the use of glucocorticoids in patients with incident rheumatoid arthritis: a population-based study. Ann Rheum Dis 2011;70:990-5.

25. Etminan M, Forooghian F, Maberley D. Inflammatory ocular adverse events with the use of oral bisphosphonates: a retrospective cohort study. Can Med Assoc J 2012;184:E431-E434.

26. Aviña-Zubieta JA, Vostretsova K, De Vera MA, et al. The risk of pulmonary embolism and deep venous thrombosis in systemic lupus erythematosus: a general population-based study. Semin Arthritis Rheum 2015;45:195-201.

27. Bernatsky S, Linehan T, Hanly JG. The accuracy of administrative data diagnoses of systemic autoimmune rheumatic diseases. $J$ Rheumatol 2011;38:1612-6.

28. Dillman DA. Mail and Internet surveys: the tailored design method. 2nd edUpdate with new internet, visual, and mixed-mode guide. Hoboken, NJ : Wiley, 20072007.

29. Guo Y, Kopec JA, Cibere J, et al. Population Survey features and response rates: a Randomized Experiment. Am J Public Health 2016;106:1422-6.

30. Becker R, Mehlkop G. Effects of Prepaid Monetary Incentives on Mail Survey Response Rates and on Self-reporting about Delinquency Empirical findings. Bull of Sociol Methodol MxO0E9thodologie Sociol 2011;111:5-25.

31. Béland Y. Canadian community health survey--methodological overview. Health Rep 2002;13:9-14.

32. EuroQol.http://www.euroqol.org/

33. Xie F, Pullenayegum E, Gaebel K, et al. A Time Trade-off-derived value set of the EQ-5D-5L for Canada. Med Care 2016;54:98-105.

34. Fries JF, Spitz P, Kraines RG, et al. Measurement of patient outcome in arthritis. Arthritis Rheum 1980;23:137-45.

35. Reilly MC, Zbrozek AS, Dukes EM. The validity and reproducibility of a work productivity and activity impairment instrument. Pharmacoeconomics 1993;4:353-65.

36. Zhang W, Bansback N, Boonen A, et al. Development of a composite questionnaire, the valuation of lost productivity, to value productivity losses: application in rheumatoid arthritis. Value Health 2012;15:46-54.

37. Zhang W, Bansback N, Kopec J, et al. Measuring time input loss among patients with rheumatoid arthritis: validity and reliability of the valuation of Lost Productivity questionnaire. J Occup Environ Med 2011;53:530-6.

38. Zhang W, Bansback N, Sun $\mathrm{H}$, et al. Estimating the monetary value of the annual productivity gained in patients with early rheumatoid arthritis receiving etanercept plus methotrexate: interim results from the PRIZE study. RMD Open 2015;1:e000042-

39. Sadatsafavi M, Rousseau R, Chen W, et al. The preventable burden of productivity loss due to suboptimal asthma control: a populationbased study. Chest 2014;145:787.

40. Connor Gorber S, Shields M, Tremblay MS, et al. The feasibility of establishing correction factors to adjust self-reported estimates of obesity. Health Rep 2008;19:71-82.

41. Stommel M, Schoenborn CA. Accuracy and usefulness of BMI measures based on self-reported weight and height: findings from the NHANES \& NHIS 2001-2006. BMC Public Health 2009;9:421.

42. Connor Gorber S, Tremblay M, Moher D, et al. A comparison of direct vs. self-report measures for assessing height, weight and body mass index: a systematic review. Obes Rev 2007;8:307-26.

43. Wong SL, Shields M, Leatherdale S, et al. Assessment of validity of self-reported smoking status. Health Rep 2012;23:47-53.

44. Elgar FJ, Stewart JM. Validity of self-report screening for overweight and obesity. evidence from the Canadian Community Health Survey. Can J Public Health 2008;99:423-7.

45. Zhang W, Bansback N, Boonen A, et al. Validity of the work productivity and activity impairment questionnaire--general health version in patients with rheumatoid arthritis. Arthritis Res Ther 2010;12:R177.

46. Gordon C, Isenberg D, Lerstrøm K, et al. The substantial burden of systemic lupus erythematosus on the productivity and careers of patients: a european patient-driven online survey. Rheumatology 2013;52:2292-301. 